\title{
Un cas d'infection acquise à Babesia microti au Canada
}

\author{
Jiayu Yang MD, Catherine Smith MD, Anthony Battad MD MSc MSP
}

- Citation : CMAJ 2021 August 9;193:E1213-7. doi : 10.1503/cmaj.201983-f

Voir la version anglaise de l'article ici : www.cmaj.ca/lookup/doi/10.1503/cmaj.201983

E n août 2019, un homme de 75 ans a consulté à l'urgence à Winnipeg parce qu'il avait de la fièvre, une myalgie et une léthargie et avait perdu $7 \mathrm{~kg}$ depuis 6 semaines. Après 3 semaines de symptômes, il avait vu son médecin de famille, qui lui avait prescrit un cycle d'une semaine d'amoxicilline avec acide clavulanique. Comme les symptômes persistaient malgré l'antibiothérapie, il a consulté dans un service d'urgence communautaire, où le médecin a constaté une anémie et une thrombocytopénie (tableau 1). Puis il a subi une tomodensitométrie (TDM) thoracique, abdominale et pelvienne en urgence dans une clinique externe, qui n'a révélé aucune particularité. Le patient a ensuite été vu à notre service d'urgence avec des symptômes persistants, et nous l'avons hospitalisé pour effectuer des examens approfondis.

Le patient s'est rappelé avoir commencé à se sentir mal alors qu'il naviguait sur le lac Pelican à Ninette, au Manitoba. Il avait alors remarqué l'apparition d'une lésion rouge de la grosseur du pouce sur sa jambe droite. Il n'avait pas vu de tiques ni d'autres lésions cutanées sur son corps. On notera que le patient avait séjourné en Arizona 6 mois avant l'apparition des symptômes et qu'il n'avait pas voyagé à l'extérieur de l'Amérique du Nord. Onze semaines avant le déclenchement des symptômes, il avait campé dans le parc provincial de Birds Hill au Manitoba. Sept semaines avant le début des symptômes, il était allé à Burlington, en Ontario (figure 1). Il avait aussi donné du sang 7 semaines avant de consulter.

À l'admission, le patient faisait de la fièvre $\left(38,6^{\circ} \mathrm{C}\right)$ et était tachycarde (105 battements/minute). L'examen physique s'est révélé sans particularités : aucun signe de piqûre d'insectes, d'éruption cutanée, de lymphadénopathie, d'hépatosplénomégalie ni d'ictère. Étant donné le résultat négatif à la TDM récente et les symptômes non spécifiques, nous avons supposé une étiologie infectieuse, et notre diagnostic différentiel s'est porté vers des infections virales atypiques.

Les analyses ont montré que la pancytopénie du patient avait progressé depuis sa dernière consultation à l'urgence, et que ses concentrations d'enzymes hépatiques étaient élevées. Les analyses de laboratoire présentées au tableau 1 montraient des signes d'hémolyse et d'insuffisance rénale aiguë. L'hémoculture, la culture d'urine et les analyses sérologiques

\section{Points clés}

- Babesia microti est transmis par Ixodes scapularis et est la principale cause de babésiose en Amérique du Nord.

- La babésiose s'accompagne généralement d'une fièvre, d'un malaise, d'une anémie et d'une thrombocytopénie intermittents, et peut coexister avec d'autres infections transmises par la tique.

- La babésiose peut ressembler au paludisme sur les frottis sanguins. Les frottis minces et la réaction en chaîne de la polymérase permettent de confirmer le diagnostic.

- Le territoire d'l. scapularis s'est étendu au Canada. Les maladies transmises par les tiques, dont la babésiose, devraient être incluses dans le diagnostic différentiel au Canada, et le traitement empirique est à envisager.

de dépistage des virus des hépatites $A, B$ et $\mathrm{C}$, du VIH, du cytomégalovirus, du virus d'Epstein-Barr, du virus du Nil occidental et de Borrelia burgdorferi étaient toutes négatives. Nous n'avons pas fait de test pour l'anaplasmose à ce moment. Sa radiographie thoracique était sans particularités.

Pour tenter d'expliquer l'anémie et la thrombocytopénie du patient, nous avons demandé un frottis de sang périphérique afin de pouvoir écarter un diagnostic d'anémie microangiopathique; l'analyse a révélé des formes intraérythrocytaires évocatrices du paludisme. Nous avons alors demandé un examen de frottis mince et de goutte épaisse. La charge parasitaire s'élevait initialement à $0,2 \%$, puis a culminé à $0,8 \%$. Comme le patient semble avoir été piqué par un insecte, mais n'a pas séjourné dans une région touchée par un paludisme endémique, nous avons soupçonné la babésiose et commencé un traitement empirique à l'atovaquone et à l'azithromycine. Dès le troisième jour de traitement, la fièvre du patient était tombée ${ }^{3}$.

L'examen poussé du frottis de sang périphérique en hématopathologie est venu appuyer le diagnostic de babésiose (figure 2 et figure 3), finalement attribué à Babesia microti au moyen d'un test par réaction en chaîne de la polymérase (PCR). La parasitémie du patient était de $0,1 \%$ au moment du congé, 5 jours après l'admission, et de $0 \%$ une semaine plus 
Tableau 1 : Résultats des analyses de laboratoire chez un homme de 75 ans atteint de babésiose

\begin{tabular}{|c|c|c|c|c|}
\hline Test & $\begin{array}{l}1 \text { semaine avant } \\
\text { l'admission }\end{array}$ & À l'admission & $\begin{array}{c}1 \text { semaine après } \\
\text { le congé }\end{array}$ & Plage de référence \\
\hline Hémoglobine (g/L) & 119 & 95 & 95 & $140-180$ \\
\hline Numération plaquettaire $\left(\times 10^{9} / \mathrm{L}\right)$ & 265 & 67 & 283 & $140-440$ \\
\hline Urée (mmol/L) & 7,9 & 11,2 & 6,0 & $2,8-7,1$ \\
\hline Aspartate aminotransférase (U/L) & 28 & 81 & 28 & $10-32$ \\
\hline Alanine aminotransférase (U/L) & 24 & 66 & 39 & $<30$ \\
\hline Phosphatase alcaline (U/L) & & 79 & & $30-120$ \\
\hline Y-glutamyl transférase (U/L) & & 54 & 60 & $5-38$ \\
\hline Lacticodéshydrogénase (U/L) & & 603 & 79 & $120-230$ \\
\hline Rapport international normalisé & & 1,1 & & $0,9-1,1$ \\
\hline Haptoglobine (g/L) & & $<0,1$ & & $0,2-2,0$ \\
\hline
\end{tabular}

tard. Sa fonction rénale a continué de s'améliorer, et sa pancytopénie et les anomalies des enzymes hépatiques sont rentrées dans l'ordre (tableau 1).

Nous avons contacté la société canadienne du sang, qui a analysé par PCR le sang donné par le patient et confirmé la présence de B. microti.

\section{Discussion}

\section{Épidémiologie de la babésiose au Canada}

La babésiose est causée par un protozoaire intraérythrocytaire appartenant au genre Babesia, transmis principalement par les tiques du genre Ixodes et parfois lors de transfusions sanguines, lors de greffes d'organe ou par voie transplacentaire ${ }^{1}$. Même si de nombreuses espèces du genre Babesia causent des infections chez l'être humain, la plupart des cas de babésiose en Amérique du Nord sont causés par $B$. microti, une espèce transmise par la tique I. scapularis, endémique dans le nord-est et le nord du midwest des États-Unis. Dans ces régions, l'incidence de la babésiose est d'environ 2000 cas par année (figure 4; voir aussi : https://www.cdc.gov/ parasites/babesiosis/resources/babesiosis_ surveillance_summary_2018.pdf) ${ }^{1}$.

Jusqu'à récemment, la babésiose

Figure 1 : Cours des événements et évolution clinique chez un homme de 75 ans atteint de babésiose. 
séroprévalence effectuée sur des dons de sang en 2013 n'avait trouvé aucun échantillon porteur d'anticorps anti-B. microti sur près de 14000 échantillons testés ${ }^{2}$. Toutefois, avec les changements climatiques, la tique $I$. scapularis a migré vers le nord et est désormais présente au Manitoba, en Ontario, au Québec, en Nouvelle-Écosse et au NouveauBrunswick ${ }^{3}$. Une autre étude de séroprévalence, menée en

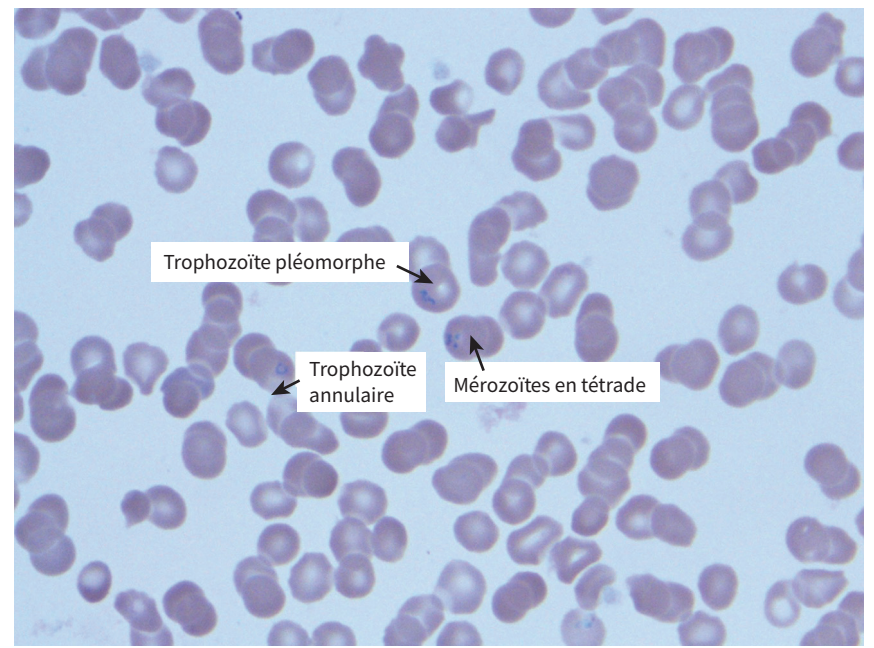

Figure 2 : Frottis sanguin d'un homme de 75 ans atteint de babésiose, montrant des trophozoïtes pléomorphes et annulaires ainsi que des mérozoïtes en tétrade, ou « en croix de Malte».
2018, a vérifié la présence de $B$. microti par TMA (transcription-mediated amplification) dans 50752 échantillons. Un seul résultat positif a été enregistré (provenant du Manitoba). Un sous-ensemble aléatoire de 14758 échantillons négatifs à la détection par TMA a été soumis au dépistage de l'immunoglobuline $\mathrm{G}$ anti-Babesia, qui a généré 4 résultats positifs (les 4 échantillons provenaient du sud-ouest de

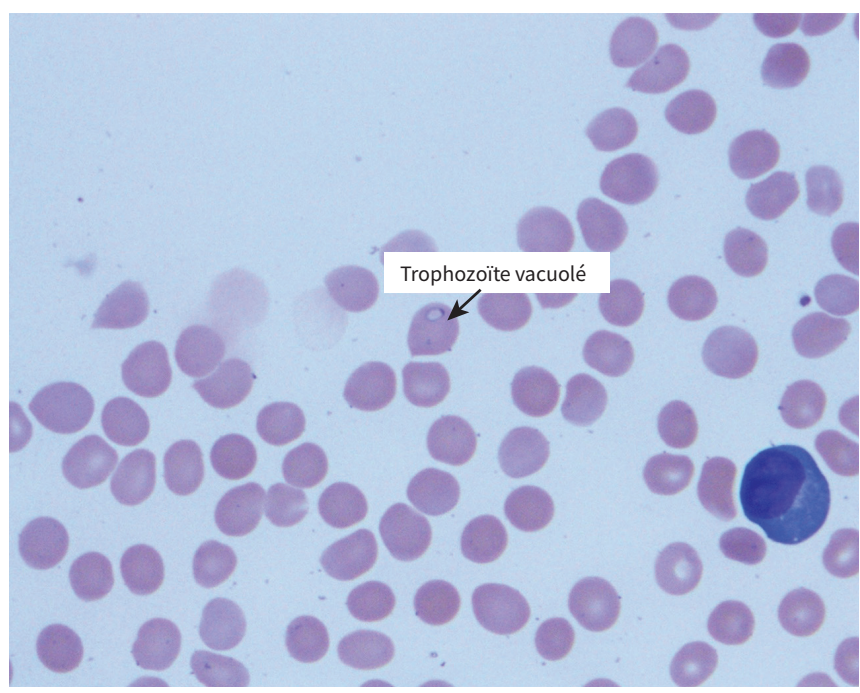

Figure 3 : Frottis sanguin d'un homme de 75 ans atteint de babésiose, montrant des trophozoïtes vacuolés.

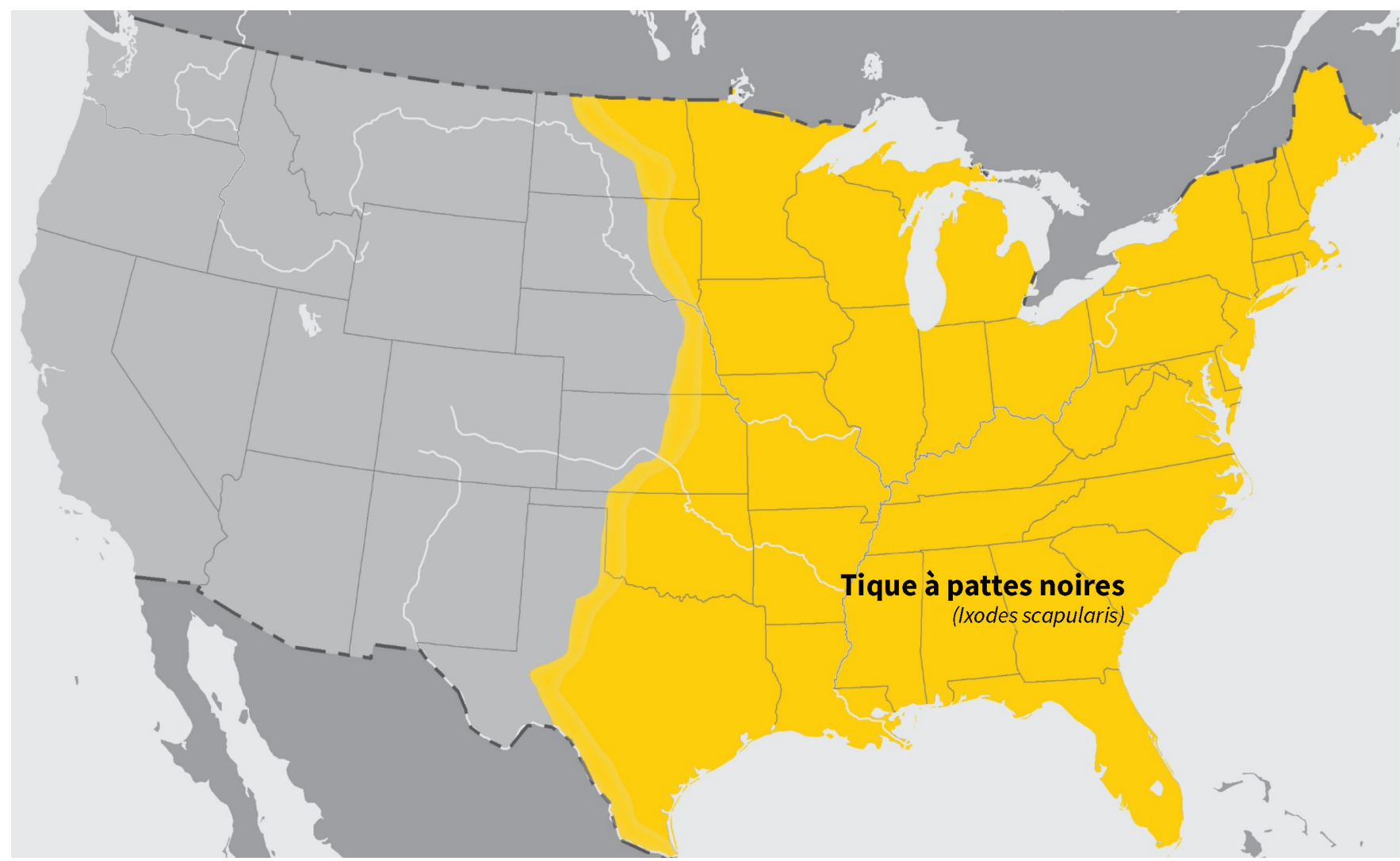

Figure 4 : Distribution approximative d'Ixodes scapularis (tique à pattes noires) aux États-Unis en 2018. (Illustration des CDC [Centers for Disease Control and Prevention]. L'utilisation de cette illustration ne signifie pas que les CDC entérinent l'article. Document par ailleurs disponible gratuitement sur le site Web des CDC.) 
l'Ontario). Un seul des 5 donneurs se rappelait avoir présenté des symptômes pseudogrippaux, 1 mois après le don ${ }^{4}$.

Le premier cas de babésiose contractée au Manitoba a été déclaré en 2013 : l'infection à $B$. microti a été confirmée par PCR chez un garçon de 7 ans qui présentait des antécédents médicaux complexes, dont une asplénie secondaire à une malrotation intestinale ${ }^{5}$. Un autre cas probable a été signalé au Manitoba en 2016, la seule province canadienne où cette maladie est à déclaration obligatoire (elle ne l'est pas non plus dans les territoires) ${ }^{6}$. Depuis, un autre cas de babésiose, causé par $B$. duncani, a été observé chez un homme de 70 ans du sud de l'Ontario après une morsure de nymphe d'l. scapularis, en $2017^{7}$.

\section{Transmission et tableau clinique}

En Amérique du Nord, le principal vecteur de B. microti est la tique $I$. scapularis, active de la fin du printemps au début de l'automne. La souris à pattes blanches, Peromyscus leucopus, en est le réservoir principal. Ixodes scapularis se nourrit du sang du cerf de Virginie, Odocoileus virginianus. L'animal ne contracte pas l'infection, mais fait proliférer la population d'l. scapularis ${ }^{1}$. La période d'incubation est en général de 1-6 semaines après la piqûre.

À l'instar du genre Plasmodium, Babesia infecte les érythrocytes en produisant des mérozoïtes, qui entraînent une hémolyse et les complications cliniques associées. Le spectre de la babésiose va d'asymptomatique à grave et fatale. L'infection asymptomatique est fréquente, comme l'a démontré une étude prospective échelonnée sur 10 ans qui a observé le phénomène chez environ le quart des adultes et la moitié des enfants infectés ${ }^{8}$. Toutefois, des manifestations cliniques ont été documentées chez des patients auparavant asymptomatiques, après une splénectomie ou une immunosuppression ${ }^{9}$. La fièvre est un des caractéristiques principales de la babésiose. Les patients présentent en général de manière sporadique fièvre, frissons, diaphorèse, céphalées, myalgie et anorexie, de même que malaise et fatigue progressifs. L'examen physique et les analyses de laboratoire montrent des caractéristiques de la pathogenèse, soit pâleur, ictère, hépatosplénomégalie, anémie hémolytique, thrombocytopénie et hausse des taux de créatinine et d'enzymes hépatiques. La maladie grave survient souvent chez les patients immunodéprimés, particulièrement chez ceux qui sont âgés, et elle s'accompagne de manifestations d'anémie grave comme un syndrome de détresse respiratoire aiguë, une coagulation intravasculaire disséminée, une insuffisance cardiaque congestive, une insuffisance rénale, un choc ou la mort. Des taux de mortalité atteignant $20 \%$ ont été signalés dans les cas graves de babésiose ${ }^{1}$.

\section{Diagnostic}

La babésiose peut être diagnostiquée au moyen de frottis sanguins minces avec coloration de Giemsa et de Wright. Les formes les plus fréquentes du parasite sont annulaires et sont souvent confondues avec les trophozoïtes de Plasmodium falciparum. Toutefois, Babesia se distingue de $P$. falciparum par la forme de ses mérozoïtes. Le parasite est appelé sporozoïte lorsqu'il pénètre les érythrocytes. À l'intérieur de la cellule, il se transforme en trophozoïte puis mue en mérozoïte, la tétrade (en «croix de Malte ») pathognomonique de l'infection (figure 1$)^{1,10}$. La réaction en chaîne de la polymérase est un test plus sensible que le frottis sanguin, et permet d'identifier l'espèce précise de Babesia en plus de confirmer le diagnostic $^{1,10}$. La sérologie de Babesia peut aussi aider au diagnostic, un titre d'anticorps de 1:1024 ou plus évoquant une infection récente ou active, même si les taux sériques doivent quadrupler entre la phase aiguë et de la phase convalescente pour confirmer l'infection ${ }^{1,10}$.

I. scapularis transmet d'autres agents pathogènes, dont les espèces du genre Borrelia, Anaplasma phagocytophilum, les espèces du genre Ehrlichia et le virus Powassan. En 2016, une analyse a révélé que des co-infections s'observent chez $1 \%$ à $28 \%$ des tiques du genre Ixodes dans les régions d'Amérique du Nord où la maladie de Lyme est endémique ${ }^{11}$. La coinfection la plus souvent décrite est celle à $B$. burgdorferi et $B$. microti, observée chez $6 \%-23 \%$ des patients atteints de babésiose ${ }^{11}$. Il est donc important de faire le dépistage d'une co-infection chez tout patient atteint de babésiose.

\section{Traitement}

Le traitement recommandé pour la babésiose est la prise orale de $750 \mathrm{mg}$ d'atovaquone toutes les 12 heures combinée à la prise orale de 500-1000 mg puis de 250-500 mg d'azithromycine 1 fois par jour pendant 7-10 jours. Il est également possible d'administrer de la clindamycine à raison de 300-600 mg par voie intraveineuse toutes les 6 heures ou de prendre $600 \mathrm{mg}$ par voie orale toutes les 8 heures et de prendre $650 \mathrm{mg}$ de quinine par voie orale toutes les 6-8 heures ${ }^{10}$. Ce traitement, associé à plusieurs effets indésirables, est toutefois recommandé dans les cas graves ${ }^{1}$. Chez les patients immunodéprimés, le traitement doit se prolonger au-delà de 6 semaines, et au moins 2 semaines après l'obtention de résultats négatifs au frottis sanguin ${ }^{10}$. Chez les patients qui souffrent de babésiose grave, avec une parasitémie de $10 \%$ ou plus, une hémolyse grave, ou une insuffisance respiratoire, rénale ou hépatique, l'exsanguinotransfusion est à envisager ${ }^{10}$.

\section{Conclusion}

Nous avons présenté ici un cas d'infection à $B$. microti chez un adulte immunocompétent, acquise fort probablement au Manitoba, au Canada, si l'on se fie à la période d'incubation typique de 1-6 semaines, mais qui pourrait aussi avoir été contractée en Ontario. Il est peu probable que le patient ait été infecté lors de son voyage en Arizona, puisque I. scapularis n'y est pas endémique, et quoique possible, une période d'incubation aussi longue ne serait pas typique. Avec les changements climatiques et l'expansion du territoire d'l. scapularis, l'incidence des maladies transmises par les tiques, dont la babésiose, est en hausse au Canada ${ }^{2,3}$. Il est donc important d'inclure la babésiose dans le diagnostic différentiel chez les patients qui présentent de la fièvre, surtout en été, même s'ils n'ont pas voyagé à l'extérieur du Canada. La découverte de 
parasites intraérythrocytaires chez un patient qui n'a pas voyagé dans des régions où le paludisme est endémique devrait éveiller des soupçons à l'égard de la babésiose et justifie un traitement empirique. Il faudrait que la déclaration aux instances de santé publique devienne obligatoire partout au Canada pour améliorer la description des cas, suivre la prévalence et mieux faire connaître cette maladie dans la communauté médicale.

\section{Références}

1. Krause PJ. Human babesiosis. Int J Parasitol 2019;49:165-74

2. O'Brien SF, Delage G, Scalia V, et al. Seroprevalence of Babesia microti infection in Canadian blood donors. Transfusion 2016;56:237-43.

3. Bouchard C, Dibernardo A, Koffi J, et al. Increased risk of tick-borne diseases with climate and environmental changes. Can Commun Dis Rep 2019;45:83-9.

4. Tonnetti L, O'Brien SF, Gregoire Y, et al. Prevalence of Babesia in Canadian blood donors: June-October 2018. Transfusion 2019;59:3171-6.
5. Bullard JMP, Ahsanuddin AN, Perry AM, et al. The first case of locally acquired tick-borne Babesia microti infection in Canada. Can J Infect Dis Med Microbiol 2014;25:e87-9.

6. Tick-borne diseases. Manitoba: Manitoba Public Health; 2018. Accessible ici : https://www.gov.mb.ca/health/publichealth/cdc/tickborne/ (consulté le 27 août 2019).

7. Scott JD. First record of locally acquired human babesiosis in Canada caused by Babesia duncani: a case report. SAGE Open Med Case Rep 2017;5: $2050313 X 17725645$.

8. Krause PJ, McKay K, Gadbaw J, et al. Increasing health burden of human babesiosis in endemic sites. Am J Trop Med Hyg 2003;68:431-6.

9. Wormser GP, Lombardo G, Silverblatt F, et al. Babesiosis as a cause of fever in patients undergoing a splenectomy. Am Surg 2011;77:345-7.

10. Wormser GP, Dattwyler RJ, Shapiro ED, et al. The clinical assessment, treat ment, and prevention of Lyme disease, human granulocytic anaplasmosis, and babesiosis: clinical practice guidelines by the Infectious Diseases Society of America. Clin Infect Dis 2006;43:1089-134.

11. Diuk-Wasser MA, Vannier E, Krause PJ. Coinfection by the tick-borne pathogens Babesia microti and Borrelia burgdorferi: ecological, epidemiological and clinical consequences. Trends Parasitol 2016;32:30-42.
Intérêts concurrents : Aucun déclaré.

Cet article a été évalué par des pairs.

Les auteurs ont obtenu le consentement du patient.

Affiliations : Département de médecine interne (Yang, Battad), Section d'infectiologie, Département de médecine interne (Smith), Université du Manitoba, Winnipeg, Man.

Collaborateurs : Tous les auteurs ont contribué à l'élaboration et à la conception des travaux. Jiayu Yang a rédigé le manuscrit. Catherine Smith et Anthony Battad l'ont révisé de façon critique pour le contenu intellectuel important. Tous ont aussi approuvé la version finale pour publication et accepté d'assumer l'entière responsabilité de tous les aspects du travail.

Propriété intellectuelle du contenu : Il s'agit d'un article en libre accès distribué conformément aux modalités de la licence Creative Commons Attribution (CC BY-NC-ND 4.0), qui permet l'utilisation, la diffusion et la reproduction dans tout médium à la condition que la publication originale soit adéquatement citée, que l'utilisation se fasse à des fins non commerciales (c.-à-d., recherche ou éducation) et qu'aucune modification ni adaptation n'y soit apportée. Voir : https://creativecommons. org/licenses/by-nc-nd/4.0/deed.fr.

Correspondance : Jiayu Yang, yangj326@myumanitoba.ca 